\title{
Determination of potassium by the use of new calibration approach in potentiometric measurements
}

\author{
Marek Dębosz $^{1}$ (D) $\cdot$ Marcin Wieczorek ${ }^{1} \cdot$ Jan Migdalski ${ }^{2} \cdot$ Paweł Kościelniak $^{1}$
}

Received: 31 March 2020 / Accepted: 14 May 2020 / Published online: 25 May 2020

(c) The Author(s) 2020

\begin{abstract}
The implementation of a new calibration approach, termed the Signal Increment Standard Addition Method (SI-SAM), to the potentiometric determination of potassium in the synthetic samples and certified reference materials, is investigated. SI-SAM is a modification of SAM that is based on the change in the way in which the extrapolation is carried out. The calibration graph is extrapolated to the intersection with the signal of the first standard solution, not to the abscissa. Potentiometry is the method of choice due to its simplicity, rapid measurements, wide linear range and fulfilment of green analytical principles. However, careful attention is required to the calibration procedure because various versions of the standard addition method, which are commonly used in potentiometry, may create some problems leading to serious systematic analytical errors. The present work proves that SI-SAM allows for the elimination of this drawback and, consequently, for the determination of the considered analyte with improved results, especially in terms of accuracy and precision.
\end{abstract}

\section{Graphic abstract}

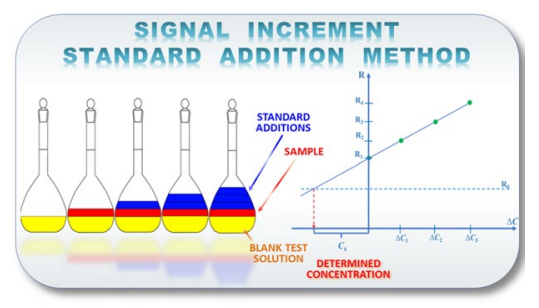

Keywords Potassium $\cdot$ Calibration method $\cdot$ Water analysis $\cdot$ Potentiometry $\cdot$ Ion selective electrode

\section{Introduction}

An analytical method described as a simple, fast, low-cost and sensitive is particularly welcomed. Potentiometry might be recommended as one of such methods, among a variety of others. In addition, the potentiometry offers several advantages, for instance it is non-destructive, may be easily

Marek Dębosz

marek.debosz@doctoral.uj.edu.pl

1 Department of Analytical Chemistry, Faculty of Chemistry, Jagiellonian University, Kraków, Poland

2 Department of Analytical Chemistry, Faculty of Materials Science and Ceramics, AGH-University of Science and Technology, Kraków, Poland automated and requires limited, if any, sample pre-treatment [1]. A group of selective potentiometric devices, namely ion selective electrodes, are widely employed in the environmental analyses. Nevertheless, although being quite popular, they are faced with a challenge of being susceptible to the interferences [2]. This situation occurs when, for instance, different levels of complexing equilibria or poisoning effect of colloidal electrolytes in the matrix are associated with the analysed sample. One way of managing this problem is to add the ionic strength adjuster to both the sample and the standard solution. Forcing conditions of equal iconic strength enables the analyte to be released from complexes and allows buffering the samples with the aim of $\mathrm{pH}$ interference elimination. 
The potentiometric measurements, as most other analytical methods, require the experimental dependence of the analytical signal on the concentration of the analyte to be established. However, as mentioned, the influence of the inferences needs to be taken into account. The calibration technique may additionally adjust the results by having an impact on the better precision and accuracy through the elimination of the systematic errors. Over the last few decades, many approaches have been proposed, ranging from simple to elaborate ones, including calibration curve method (CCM), single standard addition method (S-SAM), Gran's method (multiple standard addition method, M-SAM), potentiometric titration or methods based on the mathematical models to design calibration solutions $[3,4]$. The most widely used calibration method in potentiometry is the calibration curve method (called direct potentiometry). It requires the series of standard solutions with the analyte for which value of the signal (potential) is gathered. Based on those values, the calibration curve is derived and represented as the electromotive force (EMF) functioned on of log (concentration), as given by the Nernst equation. Then, the value of the signal for the sample is taken and interpolated via calibration graph to yield the concentration of the analyte in the analysed sample. The direct potentiometry is easily carried out and it enables the determinations in the absence of the interferents. Unfortunately, it is not robust to the influence of the matrix on the registered signals. In order to level the influence of the interferents S-SAM, Gran's method or multivariate calibration methods might be used.

S-SAM is based on the registration of the EMF for the analysed sample with the subsequent addition of the fixed volume of standard solution with the EMF being reregistered for such mixture as well. The concentration of the analyte in the sample is calculated using the following equation:

$C_{0}=\frac{V_{a} c_{a}}{V_{t} 10^{-\Delta E / s}-V_{0}}$

where $C_{0}$ is the concentration of the analyte in the sample, $V_{\mathrm{a}}$ is the volume of added standard solution, $\Delta E$ is the difference between the cell voltage before and after performing the standard addition, $\mathrm{s}$ is the empirical sensitivity of the sensor, $V_{0}$ is the sample volume and $V_{\mathrm{t}}=V_{0}+V_{\mathrm{a}}$. As may be noted, the empirical sensitivity of the electrode ( $\mathrm{mV} /$ decade of concentration) needs to be known. Moreover, the main disadvantage of this method is low precision of the results (since the measurements yield only two signals).

To overcome this issue, Gran introduced a modification based on the notion that instead of a single addition of the standard solution multiple additions should be added to the system yielding new signal with each subsequent addition [5]. The registered values of the signals are used for plotting $\left(V_{0}+V_{\mathrm{a}}\right) \cdot 10^{(\Delta E / s)}$ as a function of the added standard's volume. Through extrapolation of this curve, the volume of the standard is related to the concentration of the analyte in the analysed sample. The final result is obtained by:

$C_{0}=\frac{C_{\mathrm{a}} \cdot V_{\mathrm{x}}}{V_{0}}$

where $C_{0}$ is the concentration of the analyte in the sample, $C_{\mathrm{a}}$ is the concentration of the standard solution, $V_{\mathrm{x}}$ is the volume determined by extrapolating the calibration curve and $V_{0}$ is the initial volume of the sample solution. However, it is necessary to fulfil the requirement of keeping ionic strength constant for all the analysed solutions [3, 4].

The potentiometric titration could be listed as a fast, easy to use and easy to automate method, but with the low accuracy and lack of proper end-point indicators. The chemometric models (multivariate calibration techniques) should be noted for the cases of the analysis of multiple analytes. Among those models, class least squares (CLS), principal component regression (PCR) and partial least squares (PLS) are noted [6].

Multivariate calibration methods used sensor array analyses as well; especially when these arrays were developed as high selectivity sensors for multicomponent analysis (microchips or microarrays of DNA) or as arrays of poorly selective sensors aimed at characterizing complex properties (e.g. odours and flavours) [7].

Among other methods used for the calibration of ISE arrays, Neural Networks might be distinguished as a tool for calibration that does not require the theoretical coefficients based on Nikolsky-Eisenman expression to be known. This method allows for the separation of the responses of different ions interfering on an array of ISEs making it possible to develop ISEs for industry and environmental analyses purposes [8]. However, as noted in [6], the potentiometric data are characterised by mostly non-linear responses and this might be the reason for seldom examination of multivariate systems.

One of the most common approaches to the calibration; namely, classical standard addition method, used when multiplicative interference effect is expected, cannot be employed since the extrapolation of the calibration curve to the signal measured for the blank solution (without the analyte) may lead to the erroneous analytical results. The aforementioned extrapolation-related problem may be approached with the Signal Increment Standard Addition Method (SI-SAM). This method may be viewed as a version of the classical SAM, in which the graph is extrapolated to the intersection with the signal of first standard solution not to the abscissa axis. It has been successfully applied in the determination of $\mathrm{Cr}(\mathrm{VI})$ using electroanalytical method based on the electrostriction phenomenon. The SI-SAM enables minimizing the systematic error present in cases when 
the shape of the calibration graph in the extrapolation area is unknown or uncertain. As the SAMs version, it is also able to compensate a systematic error caused by multiplicative interference effect $[9,10]$.

In this work, the suitability of this calibration method in potentiometric measurements was verified on the example of determination of potassium in synthetic samples and certified reference materials.

\section{Results and discussion}

\section{Method}

The calibration procedure begins with the measurement of the signal $R_{0}$ for the first standard solution (so-called 'blank test solution') with optimised concentration of the analyte. Then, the appropriate volume $\Delta V_{\mathrm{s}}$ of a sample (with unknown analyte concentration) is added to the blank test solution and signal $R_{1}$ is measured. In the next step, the signals $R_{2}, \ldots R_{\mathrm{n}}$ are measured after each additions of the standard (added with appropriately increased volume) to the mixture of the sample and standard solution.

On the basis of the received data, the calibration graph is constructed as the relationship between signal $R$ and cumulative concentration $\Delta C$ of added standard (see Fig. 1). In order to obtain the analytical result, the calibration graph is extrapolated to the intersection with the signal of the blank test solution (first standard solution) and this point is projected onto abscissa axis (Fig. 1).

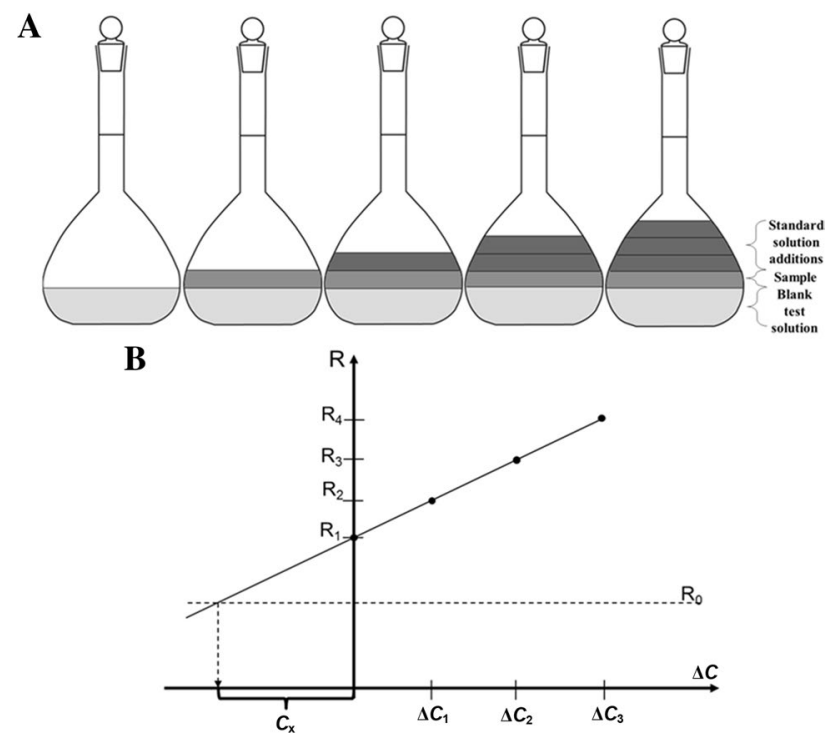

Fig. 1 The principle of the Signal Increment Standard Addition Method. a Preparation of the calibration solutions, $\mathbf{b}$ the calibration graph indicating the analytical result, $C_{\mathrm{x}}$, when extrapolated to signal $\mathrm{R}_{0}$ measured for the blank test solution

\section{Analysis}

The synthetic sample containing only $2.57 \mathrm{mmol} \mathrm{dm}{ }^{-3}$ potassium ions was analysed. The appropriate volumes of stock standard solution of potassium chloride were added during measurements. The volume of stock standard solution was chosen to obtain the growth of the signal at around $2 \mathrm{mV}$. In order to obtain satisfactory analytical results in terms of accuracy and precision, the first thing was to determine the optimal value of the concentration of the blank test solution. Figure $2 \mathrm{a}$ and $\mathrm{b}$ presents the results obtained by SI-SAM when extrapolation is carried out to the measured signal of water and to the signal of potassium chloride (with appropriately chosen concentration), respectively.

The presented data are for two independent measurements for the same analysed synthetic sample. It can be seen that the arrangement of the measured signals for situation when water plays the role of the blank test solution is non-linear. It may have been caused by the change in the ionic strength of the solution during the measurements.

It is apparent that the extrapolation area differs in both of these cases. If water plays the role of the blank test solution, the calibration graph has to be extrapolated in a definitely too large area (defined by $\Delta C<0$ ) in comparison with the experimental one $(\Delta C \geq 0$; Fig. 1a). As the linear response of working electrodes is strongly limited, the calibration graph extrapolated linearly may lead to extremely inaccurate analytical result. A way of overcoming this problem could be the sample dilution, but taking the nature of the electrodes into consideration, the obtained results would be biased due to the ion-flux membrane effect. The analyte concentration in the blank test solution should be chosen in accordance to the linear range of the electrodes in use and should be within this range. This operation leads to the decrease of the difference between experimental and extrapolation areas, which becomes acceptable (Fig. 2b) and the measurements are carried out in the known working range. It minimises the risk of a systematic error that could result from an unknown course of calibration curve in the area of its extrapolation. The analyte concentration in the standard playing the role of the blank solution was chosen to measure the signals for all calibration solutions $(\Delta C \geq 0)$ within the range of circa $5 \mathrm{mV}$.

Table 1 presents the results obtained by the use of two types of calibration methods: the calibration curve method (CCM) and SI-SAM. The concentration values are given along with $T$ test confidence interval (with 5\% significance level). In the case of a pure potassium chloride solution, calibration methods work appropriately. This means that the SI-SAM method can be utilised in potentiometric measurements. The results obtained for the synthetic samples with the addition of interferents, sodium and calcium, show that the SI-SAM delivers 
Fig. 2 The SI-SAM calibration plots for the potassium determination when water $\mathbf{a}$ and the potassium chloride solution b played a role of the blank test solution
Table 1 Results obtained in potassium determination using calibration curve method (CCM) and signal increment standard addition method (SI-SAM) with confidence interval (5\% significance level)
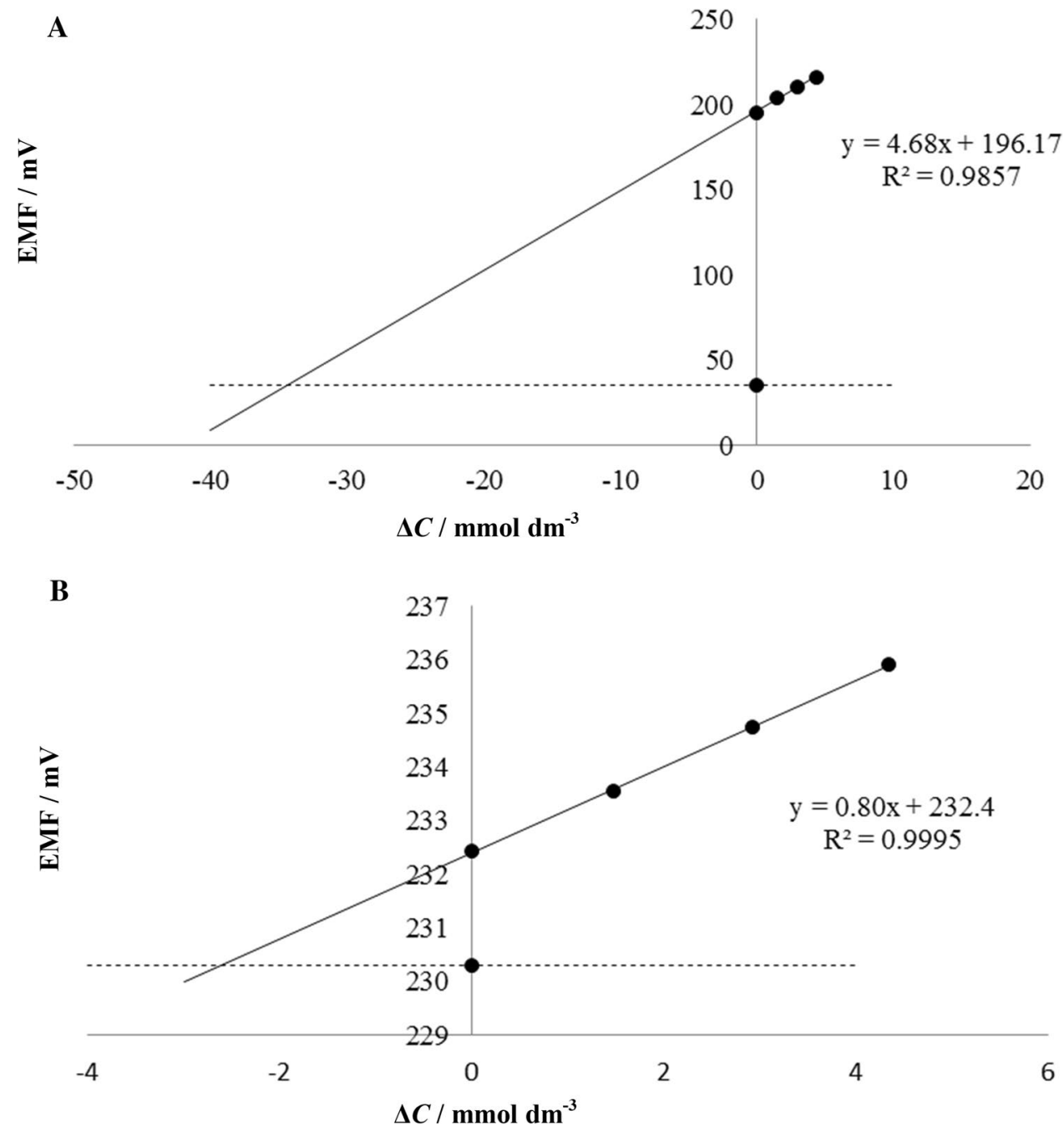

\begin{tabular}{|c|c|c|c|c|c|}
\hline \multirow[t]{2}{*}{ Sample } & \multirow[t]{2}{*}{$C_{0} / \mathrm{mmol} \mathrm{dm}^{-3}$} & \multicolumn{2}{|l|}{$\mathrm{CCM}$} & \multicolumn{2}{|l|}{ SI-SAM } \\
\hline & & $C_{\mathrm{x}} / \mathrm{mmol} \mathrm{dm}^{-3}$ & $\mathrm{RE} / \%$ & $C_{\mathrm{x}} / \mathrm{mmol} \mathrm{dm}^{-3}$ & $\mathrm{RE} / \%$ \\
\hline $\mathrm{KCl}$ & 2.57 & $2.60 \pm 0.24$ & 1.07 & $2.52 \pm 0.15$ & -1.90 \\
\hline $\mathrm{KCl}+\mathrm{NaCl}$ & & $3.56 \pm 0.52$ & 39.22 & $2.61 \pm 0.02$ & 1.63 \\
\hline $\mathrm{KCl}+\mathrm{Ca}\left(\mathrm{NO}_{3}\right)_{2}$ & & $2.45 \pm 0.27$ & -5.72 & $2.49 \pm 0.15$ & -3.18 \\
\hline $\mathrm{KCl}+\mathrm{Ca}\left(\mathrm{NO}_{3}\right)_{2}+\mathrm{NaCl}$ & & $2.48 \pm 0.27$ & -2.53 & $2.70 \pm 0.13$ & 5.14 \\
\hline Waste water-CRM & 1.02 & $1.05 \pm 0.07$ & 2.94 & $1.05 \pm 0.10$ & 2.94 \\
\hline Drinking water-CRM & 0.33 & $0.36 \pm 0.00$ & 9.76 & $0.31 \pm 0.01$ & -6.10 \\
\hline
\end{tabular}

$R E$ relative error, $C_{0}, C_{\mathrm{x}}$ expected and found analyte concentrations, respectively acceptable results and those results are better than CCM in terms of accuracy and precision. It is evident in the case of synthetic sample, when sodium ions are present along with potassium ions.

The CCM and SI-SAM methods were also used when certified reference materials were analysed for the potassium determination. The favourable values of relative errors confirm the possibility of using the proposed method as a calibration method in potentiometric measurements. The precision of the analytical results was also acceptable in all of the considered cases. 


\section{Conclusion}

The presented study shows that the developed calibration approach is an effective and helpful analytical tool and can be utilised in potentiometric measurements. Calibration by SI-SAM is not only simple and fast but, what is much more important, it delivers more accurate and precise results in comparison with CCM and does not require knowing what the empirical sensitivity of the electrode is. Moreover, as a version of SAM, it offers the possibility of reducing the systematic error caused by the multiplicative interference effect. Under well-defined instrumental conditions, it offers the determination of an analyte with improved accuracy and precision. The presented study invites further investigation, in particular in the scope of determination of other ions routinely screened for in drinking water. It would be especially interesting to investigate it in simultaneous determination of such ions with the use of flow manifold and all solid-stateion selective electrodes appropriate for this task.

\section{Experimental}

\section{Reagents and solutions}

The following chemicals purchased from Sigma-Aldrich (Germany) were used for membrane preparation: highmolecular weight poly(vinyl chloride) (PVC), bis(2-ethylhexyl)sebacate (DOS), tetrahydrofuran (THF), potassium tetrakis( $p$-chlorophenyl)borate (KTpClPB), valinomycin (potassium ionophore). Other chemicals, such as $\mathrm{KCl}$ (Chempur, Poland), $\mathrm{Ca}\left(\mathrm{NO}_{3}\right)_{2} \bullet 4 \mathrm{H}_{2} \mathrm{O}$ (POCH, Poland), $\mathrm{NaCl}$ ( $\mathrm{POCH}$, Poland), $\mathrm{CH}_{3} \mathrm{COOLi} \cdot 2 \mathrm{H}_{2} \mathrm{O}$ (Chempur, Poland) were also utilised. All chemicals were of analytical grade. The $0.1 \mathrm{~mol} \mathrm{dm}^{-3}$ lithium acetate was prepared by dissolving adequate amount of substance and was utilised as the ionic strength adjuster. Stock standard solutions of potassium chloride $\left(0.2 \mathrm{~mol} \mathrm{dm}^{-3}\right)$, calcium nitrate and sodium chloride were prepared by dissolving adequate amount of salts in $0.1 \mathrm{~mol} \mathrm{dm}^{-3}$ lithium acetate. Ultrapure water $(18.2 \mathrm{M} \Omega \mathrm{cm})$ from an HLP 5 system (Hydrolab, Poland) was used throughout the work.

\section{Preparation of solid contact potassium selective electrode}

The silver electrodes were polished with $0.3 \mu \mathrm{m}$ alumina, rinsed thoroughly with deionised water and ultrasonicated for at least $4 \mathrm{~min}$. Potassium selective electrodes were prepared on silver electrode (diameter $2 \mathrm{~mm}$ ) by drop casting method. The ion selective membrane had the following

composition: was consisted of $65.8 \%$ (w/w) plasticiser (DOS), $1 \%(\mathrm{w} / \mathrm{w})$ ionophore (Valinomycin), 33\% (w/w) polymer (PCV) and $0.2 \%(\mathrm{w} / \mathrm{w})$ ion exchanger (KTpClPB). The components were dissolved in $1 \mathrm{~cm}^{3}$ of THF to produce a solution with $15 \%$ dry weight. A $15 \mathrm{~mm}^{3}$ of the ion selective membrane cocktail was deposited by drop casting onto the electrode surface. Then, the electrodes were left overnight to ensure evaporation of THF from the membranes. Subsequently, the electrodes were conditioned in $0.1 \mathrm{~mol} \mathrm{dm}^{-3}$ potassium chloride $(\mathrm{pH}=7.0)$ for at least $24 \mathrm{~h}$. Prior to the uses of prepared electrodes for potentiometric measurements, the linear range of working electrodes was checked. In this case, the linear range was between $0.01 \mathrm{mmol} \mathrm{dm}^{-3}$ and $0.1 \mathrm{~mol} \mathrm{dm}^{-3}$.

\section{Basic analytical parameters of potassium selective electrodes}

The calibration measurements were carried out in the range of $0.01 \mathrm{mmol} \mathrm{dm}^{-3}$ and $0.1 \mathrm{~mol} \mathrm{dm}^{-3}$. The selected range matched the linear range of the utilised electrodes. The value of the slope in this concentration range was very close to theoretical Nernstian response and was equal to $56.27 \pm 0.17 \mathrm{mV} /$ decade.

The potentiometric selectivity coefficients for potassium selective electrode were determined by the separate solution method (SSM) [11] are shown in Table 2. Selectivity measurements were carried out in $0.01 \mathrm{~mol} \mathrm{dm}^{-3}$ solutions of different salts and the selectivity coefficients were calculated using the potential values for the solutions of main and interfering ion as parameters in the Nikolsky-Eisenman equation.

\section{Samples}

Four synthetic samples were analysed. The first one was a pure potassium chloride. The second and the third samples were additionally containing sodium chloride and calcium nitrate, respectively, as potential interferents. The last one was the mixture of all of those compounds. The ratio of the concentration of the determined ion to the concentration of the interfering one was 1:1. Certificate Reference Standard EnviroMAT Waste Water PlasmaCal and EnviroMAT Drinking Water EP-H-1 (SCP SCIENCE) were prepared

Table 2 Potentiometric selectivity coefficients for potassium selective electrodes

\begin{tabular}{ll}
\hline \multicolumn{2}{l}{ Selectivity coefficients } \\
\hline Interferents & $\log K_{\mathrm{K}, \mathrm{M}}{ }^{\text {pot }}$ \\
\hline $\mathrm{Na}^{+}$ & -2.60 \\
$\mathrm{Ca}^{2+}$ & -3.85 \\
$\mathrm{H}^{+}$ & -3.51 \\
$\mathrm{NH}_{4}^{+}$ & -1.87 \\
$\mathrm{Mg}^{2+}$ & -4.07 \\
\hline
\end{tabular}


by diluting them in accordance with the manufacturer's instructions. Ionic strength of all samples was stabilised with $0.1 \mathrm{~mol} \mathrm{dm}^{-3}$ lithium acetate. Each determination was repeated three times in the same instrumental conditions.

\section{Instrumentation}

All potentiometric measurements were performed using a 16-channel LawsonLab (USA) potentiometer with EMF Suite version 2.0 data logging program. Potentials were measured against a flow-through $\mathrm{Ag} \mid \mathrm{AgCl}\left(3 \mathrm{~mol} \mathrm{dm}^{-3}\right) \mathrm{KCl}$ reference electrodes obtained from Mineral, Warsaw. All potentiometric measurements were performed at $20 \pm 2{ }^{\circ} \mathrm{C}$. The measurements were carried out in the three-electrode system in quartz vessel of $25 \mathrm{~cm}^{3}$ volume covered with a PMMA cover with four holes matched the size of each electrode and an additional hole for adding standard solution. Magnetic stirrer (Wigo, Poland) was also used.

Acknowledgements M.D. has been partly supported by the EU Project POWR.03.02.00-00-I004/16.

Open Access This article is licensed under a Creative Commons Attribution 4.0 International License, which permits use, sharing, adaptation, distribution and reproduction in any medium or format, as long as you give appropriate credit to the original author(s) and the source, provide a link to the Creative Commons licence, and indicate if changes were made. The images or other third party material in this article are included in the article's Creative Commons licence, unless indicated otherwise in a credit line to the material. If material is not included in the article's Creative Commons licence and your intended use is not permitted by statutory regulation or exceeds the permitted use, you will need to obtain permission directly from the copyright holder. To view a copy of this licence, visit http://creativecommons.org/licenses/by/4.0/.

\section{References}

1. Michałowski T, Pilarski B, Ponikvar-Svet M, Asuero AG, Kukwa A, Młodzianowski J (2011) Talanta 1530:1537

2. Urbanowicz M, Pijanowska DG, Jasiński A, Bocheńska M (2018) Multianalyte calibration methods for potentiometric integrated sensors system for determination of ions concentration in a body fluids. XV International Scientific Conference on Optoelectronic and Electronic Sensors (COE)

3. Thomas JDR (2013) Ion-selective electrodes in environmental and toxicological analysis. In: Albaiges J (ed) Analytical techniques in environmental chemistry. Elsiever, London, p 543

4. Banica FG (2012) Analysis with potentiometric ion sensors. In: Chemical sensors and biosensors-Fundamentals and applications. Wiley, London, p 200

5. Gran G (1952) Analyst 661:671

6. Ni Y (1998) Anal Chim Acta 145:152

7. García-Villar N, Saurina J, Hernández-Cassou S (2001) Fresenius J Anal Chem 1001:1008

8. Baret M, Massart DL, Fabry P, Conesa F, Eichner C, Menardo C (2000) Talanta 863:877

9. Wieczorek M, Kochana J, Knihnicki P, Wapiennik K (2017) Talanta 383:390

10. Kościelniak P, Wieczorek M (2016) Anal Chim Acta 14:28

11. Umezawa $\mathrm{Y}$, Bühlmann $\mathrm{P}$, Umezawa K, Tohda K, Amemiya S (2000) Pure Appl Chem 2000:1851

Publisher's Note Springer Nature remains neutral with regard to jurisdictional claims in published maps and institutional affiliations. 